\title{
Research on the Structure of Composite Solid Propellant Cross-linked Network by Nuclear Magnetic Resonance
}

\author{
$\mathrm{Ke} \mathrm{Li}{ }^{\text {a }}$, Jian Zheng ${ }^{\mathrm{a}}$, Xiao Zhang ${ }^{\mathrm{a}}$, Yongqiang $\mathrm{Du}^{\mathrm{a}}$ \\ Ordnance Engineering College, Shijiazhuang 050003, China \\ a763722187@qq.com
}

\begin{abstract}
Keywords: composite solid propellant, nuclear magnetic resonance, transverse relaxation time, crosslink density.
\end{abstract}

\begin{abstract}
The nuclear magnetic resonance (NMR) method is introduced in order to make the test of crosslink density of composite solid propellant easier than traditional methods. The research status of mechanical properties of nuclear magnetic resonance and solid propellant is briefly introduced, so is the basic principle of crosslink density and transverse relaxation time of solid propellant. A test example is shown, and the crosslink density and transverse relaxation time of the HTPB binder sample are measured. The NMR method is expected to be a new way to evaluate the mechanical properties of propellant, which exhibits a lossless, economical and rapid advantage in NMR testing.
\end{abstract}

\section{Introduction}

Composite solid propellant is a polymer-based filler-type composite materials, which can be used as the engine power source can significantly improve the survival and combat effectiveness of weapons ${ }^{[1]}$. In the manufacture, storage, transportation and use, the propellant must have good mechanical properties to ensure that it will not cause excessive deformation or damage due to the various external loads. The crosslink network structure of the propellant affects even the physical and mechanical properties such as tensile strength, elongation at break, modulus, compression deformation, swelling rate and hardness of the propellant ${ }^{[2]}$. The structure of the micro-crosslinked network structure of the solid propellant will be reflected in the changes in mechanical properties, if you can find this relevance, and combined with the propellant damage criteria, it can be used as an important basis for assessing the effectiveness of propellant failure. The cross-linked network structure of propellant can be characterized by crosslink density and transverse relaxation time ${ }^{[3]}$. Nuclear magnetic resonance (NMR) is applied to the study of the mechanical properties of solid propellant, and to get the crosslink density and transverse relaxation time.

\section{Research status}

In 1946 the two research groups led by Prof. Bloch of Stanford University and Prof. Purecell of Harvard University conducted the first independent study with different samples (water and paraffin) and different methods (double coil induction method and single coil absorption method) Nuclear magnetic resonance (NMR) experiments ${ }^{[4-5]}$. Since then, NMR began to develop rapidly, was applied in many areas, showing a great vitality and broad application prospects. Today, NMR technology is mainly used in the following three areas ${ }^{[6]}$ : (1) In medicine, the use of proton nuclear magnetic resonance imaging (MRI) principle for clinical diagnosis of imaging, detection of body organs and tissues; (2) In biological applications, NMR can be used for the 3D structure of protein molecules, and explain its structural sequence and function of the association; (3) In chemistry, NMR is an important method for the study of polymer, glass, ceramics, resin and piezoelectric materials and so on. Cui Haibiao et al. ${ }^{[7]}$ applied D_T_2 two-dimensional nuclear magnetic resonance to logging technology which provides a new tool for logging evaluation. Li Jielin et al. ${ }^{[8]}$ studied the characteristics of rock NMR under freezing-thawing conditions, providing reliable experimental data for the study of rock freezing and thawing damage mechanisms. 
As a relatively new and advanced research method, Wang Fangfang et al. ${ }^{[9]}$ predicted that the NMR method would lead the fashion in the study of explosives; Zhao Shouyuan et al. ${ }^{[10]}$ studied the relationship between transverse relaxation time and the crosslink density by low-field solid-state NMR; Jia Lin et al. ${ }^{[11-12]}$ used low frequency NMR method to detect the curing process of binder crosslink density, providing a reference for propellant pulp curing mechanism; Wang Minchang et al. ${ }^{\text {[13] }}$ introduced the NMR method to test the composition of a certain type of liquid propellant, promoting the quantitative analysis of propellant.

In the study of composite solid propellants, the research of mechanical properties has been the most important, and the influence of cross-linked network structure has even determined its mechanical properties. The study of the cross-linked network structure of propellant is of great significance to formulation design and material performance optimization ${ }^{[2]}$. Therefore, we can also use nuclear magnetic resonance in the study of solid propellants, analyzing and explaining the propellant mechanical properties from the perspective of micro-cross-linked network structure. Although nuclear magnetic resonance is widely used in the study of polymers, the specific research on the propellant has not yet fully developed, this relatively new field of research is very promising.

\section{Fundamental}

\subsection{Transverse relaxation time $T_{2}$}

Transverse relaxation time $T_{2}$ also known as spin-spin relaxation time, is caused by the energy exchange within the spin system, reflecting the chemical environment in which the hydrogen protons in the polymer of the sample, and the hydrogen protons force and degree of freedom are closely related, while the hydrogen protons and the internal structure of the sample are inseparable ${ }^{[12]}$. The greater the restraint of hydrogen protons or the smaller the degree of freedom, the shorter is $T_{2}$. Different mechanical properties of the sample is not the same, the internal chemical environment of the molecule must be different, the hydrogen proton by the constraints or degrees of freedom are different, that is, the length of $T_{2}$ is not the same. Therefore, we can use different changes of $T_{2}$ in the relationship between the mechanical properties analogy to determine the mechanical properties of the propellant is good or bad, as Damadian ${ }^{[14]}$ identified the tumor.

The transverse relaxation time $T_{2}$ is measured with the CPMG pulse sequence ${ }^{[15]}$ (shown in Fig.1), overcoming the shortcomings of the long cycle of the spin echo sequence and the large error of the $\mathrm{CP}$ sequence, and can measure $T_{2}$ quickly and accurately.

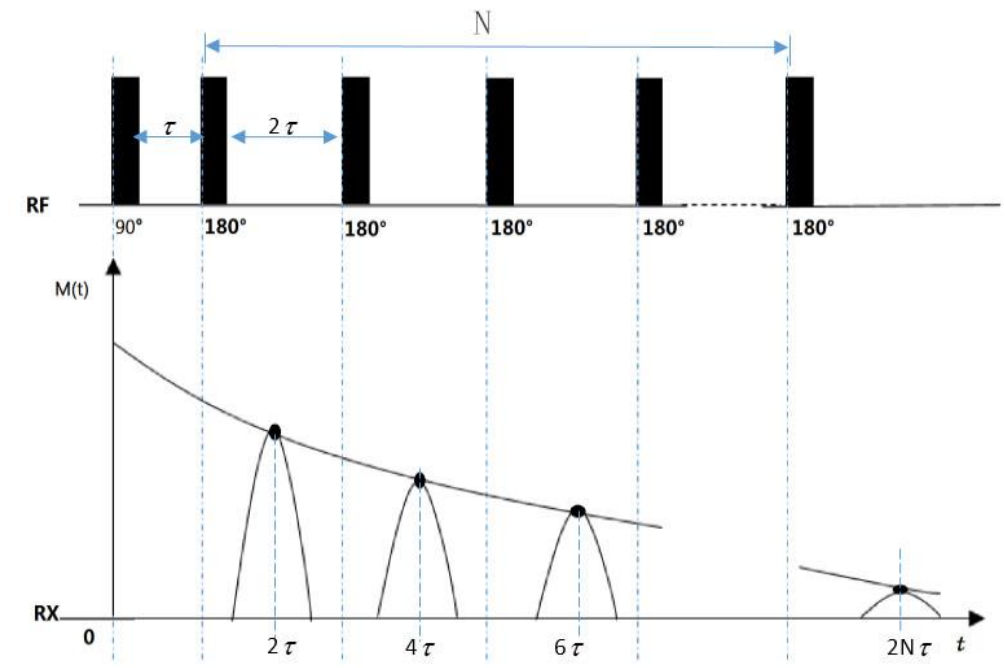

Fig.1 CPMG pulse sequence test $\mathrm{T}_{2}$

In the Figure, $\mathrm{RF}$ is the trigger time; $\mathrm{RX}$ is the reception time; $\tau$ is the half-echo time. 
After the $90^{\circ}$ RF pulse, $M(t)=M_{0}$, the nuclear spin begins to move freely and relax, and the resonant signal at this time is called the free induction decay (FID) signal ${ }^{[15]}$, as described in Equation 1:

$$
M(t)=M_{0} \times e^{-t / T_{2}}
$$

Then add $180^{\circ}$ pulse at $t=\tau, 3 \tau, 5 \tau, \cdots,(2 \mathrm{~N}-1) \tau$, then get the echo at $t=2 \tau, 4 \tau, 6 \tau, \cdots, 2 N \tau$, the peaks are:

$$
M(2 N \tau)=M_{0} \times e^{-2 N \tau / T_{2}}
$$

And then transverse relaxation time $T_{2}$ can be calculated based on these peaks .

\subsection{Crosslink density $N_{0}$}

The effect of prepolymer on the mechanical properties of propellant is mainly through the structure of solidified cross-linked network, and crosslink density is one of the most important parameters to describe the network structure. The crosslink density is a quantitative description of the degree of crosslink, defined as the concentration of the effective network chain in the unit volume, that is, the number of moles of the effective network chain per unit volume ${ }^{[1]}$. Propellant network structure (shown in Fig.2) is mainly composed of three parts: (1) cross-linked part; (2) hanging part of the chain; (3) free chain part.

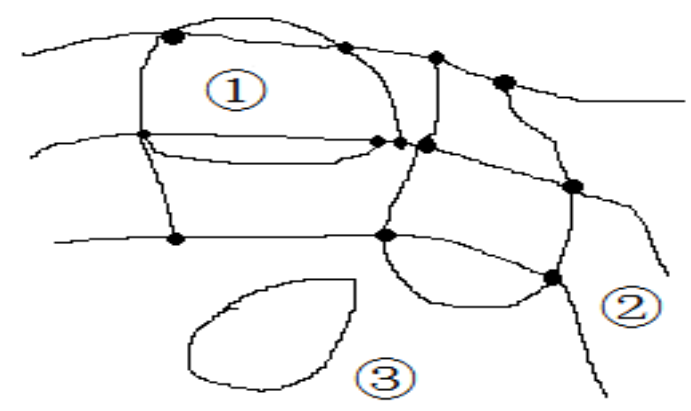

Fig.2 Propellant network structure

According to the theory of viscoelasticity of rubber, the elastic stress $\sigma$ of cross-linked rubber under uniaxial tension is proportional to the crosslink density $N_{0}{ }^{[16-17]}$,

$$
\sigma=N_{0} R T\left(\lambda-\lambda^{-2}\right)
$$

Among them $R=8.3144 \mathrm{~J} \cdot \mathrm{K}^{-1} \mathrm{~mol}^{-1}$, is the molar gas constant, $T$ is thermodynamics temperature $(\mathrm{K}), \lambda$ is deformation $\operatorname{rate}\left(\lambda=L / L_{0}=1+\varepsilon\right), N_{0}$ is crosslink density ( $\left.\mathrm{mol} / \mathrm{ml}\right), \sigma$ is elastic stress $\left(10^{-1} \mathrm{pa}\right)$.

The cross-linked structure of the polymer is divided into chemical crosslink and physical crosslink. Chemical crosslink is mainly $\mathrm{C}-\mathrm{C}$ bond and the molecular chain between the combination of physical crosslink mainly molecular chain of geometric entanglement, hydrogen bonds and Van der Waals force and so on ${ }^{[18]}$.

The chemical crosslink of the molecular chain provides good heat resistance and mechanical properties for chemically cross-linked binder-based propellants to ensure that the propellant can withstand a certain stress and strain loading and maintain a stable size. However, the high crosslink density causes the polymer to be formed to be hard and brittle, resulting in a decrease in the impact strength of the propellant, which does not meet the performance requirements of the propellant for adherent casting. In general, adherent casting propellant must have a good elasticity, requiring a low crosslink density, low modulus, high strain capacity. 


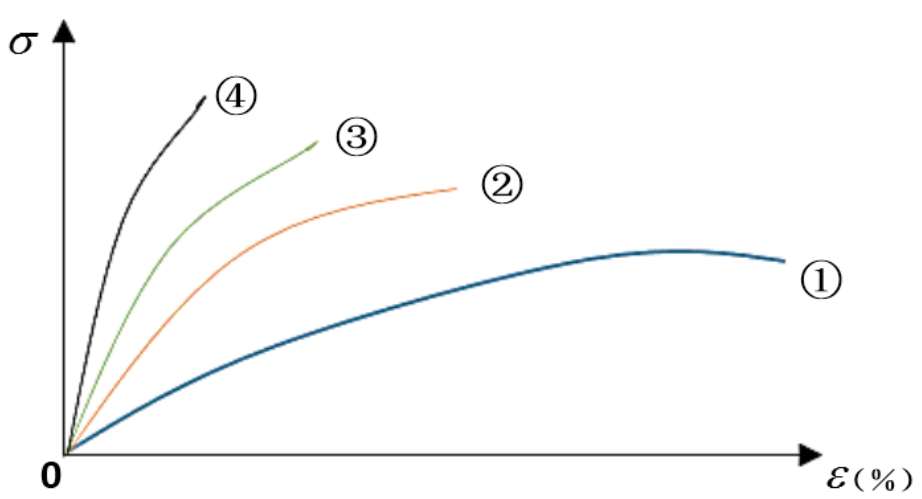

Fig.3 Relationship between crosslink density and tensile properties (crosslink density N0:1) $<$ (2) $<$ (3) < (4)

The general relationship between the crosslink density and the tensile property of the solid propellant is shown in Fig.3. The graph $\sigma$ is the stress and $\varepsilon$ is the strain of the propellant during the stretching process. It can be seen from the Figure that with the increase of the crosslink density of the propellant, the tensile strength and the elastic modulus $E$ increase with the increase of the plasticity, but the plasticity decreases and the brittleness increases more easily (the maximum strain $\varepsilon_{\max }$ becomes lower)

The methods for testing the crosslink density of propellants are swelling method ${ }^{[19-20]}$, rheological method ${ }^{[21]}$, equilibrium modulus method ${ }^{[22]}$, stress-strain method ${ }^{[23]}$ and NMR method ${ }^{[24]}$. The traditional method of testing has been very mature ${ }^{[25]}$, and as a new method, nuclear magnetic resonance method has advantages of short time, the wide temperature range, the results reproduce well, provide information and other characteristics, and can distinguish between physical and chemical crosslink that other methods can not do ${ }^{[2]}$. Zhao Fei et al. ${ }^{[26]}$ demonstrated that the crosslink density measured by NMR method has a good correlation with the results obtained by swelling method, and illustrated the feasibility of nuclear magnetic resonance method.

When measures crosslink density by NMR, the total magnetization decay process and crosslink part and hanging part of the chain are related to the following functional equation :

$$
M(t)=A \times \exp \left(-\frac{t}{T_{21}}-0.5 \times q \times M r l \times t^{2}\right)+B \times \exp \left(-\frac{t}{T_{22}}\right)+A_{0}
$$

The meaning of the parameters: $M(t)$ represents the magnetization intensity vector; $A$ represents the total signal ratio $(\%)$ of the cross-linked portion (chemical crosslink and physical crosslink) signals; $T_{21}$ represents the transverse relaxation time of the cross-linked portion(ms); $q$ represents the anisotropy rate of the cross-linked moiety, which is the ratio of the residual dipole moment to the residual dipole moment below the glass transition temperature at the sample test temperature, $q$ reflects the degree of molecular binding from the nuclear magnetic angle, the larger the cross linking density is, the greater the $q$ will be, indicating the greater the degree of binding to the molecule; $\mathrm{Mrl}$ represents the residual dipole moment of the sample below the glass transition temperature $\left(10^{-4} \mathrm{~s}^{-2}\right) ; B$ represents the proportion of the entire signal of the hanging chain part of the $\operatorname{signal}(\%) ; T_{22}$ represents the transverse relaxation time of the hanging tail chain portion $(\mathrm{ms}) ; A_{0}$ is a fitting parameter, no physical meaning.

The crosslink density is usually calculated using equation (5):

$$
N_{0}=\frac{\rho}{M_{C}}
$$

$N_{0}$ is the crosslink density of the sample; $\rho$ is the sample density $(\mathrm{g} / \mathrm{ml}) ; M_{C}$ is the average number of samples, that is, the molecular weight between adjacent crosslink points $(\mathrm{kg} / \mathrm{mol})$.

Bring (5) into equation (6): 


$$
M_{c}=\frac{3 C M_{r u}}{5 N \sqrt{q}}
$$

The equation for simplifying the crosslink density is (7):

$$
N_{0}=\frac{5 \rho N \sqrt{q}}{6 C M_{r u}}
$$

$N$ is the number of main chain bonds of the repeating unit; $C$ is the number of bonds in the main chain in the statistical segment; $M_{r u}$ is the molar mass of the repeating unit.

\section{Test example}

Equipment: VTMR20-010V-T nuclear magnetic resonance temperature analysis system, magnetic field strength $0.5 \pm 0.05 \mathrm{~T}$, probe coil $\Phi 10 \mathrm{~mm}$, temperature range of $30 \sim 130{ }^{\circ} \mathrm{C}\left( \pm 0.3{ }^{\circ} \mathrm{C}\right)$.

Sample: Hydroxyl-terminated polybutadiene (HTPB) binder, density of $0.7 \mathrm{~g} / \mathrm{ml}$, curing in the test tube $(\Phi 8.5 \mathrm{~mm} \times \mathrm{H} 20 \mathrm{~mm})$ with the equipment.

The sample was placed in a $30^{\circ} \mathrm{C}$ incubator for 20 minutes and placed in a nuclear magnetic resonance apparatus. The test temperature was $30^{\circ} \mathrm{C}$. Collect the signal spectrum (shown in Fig. 4):

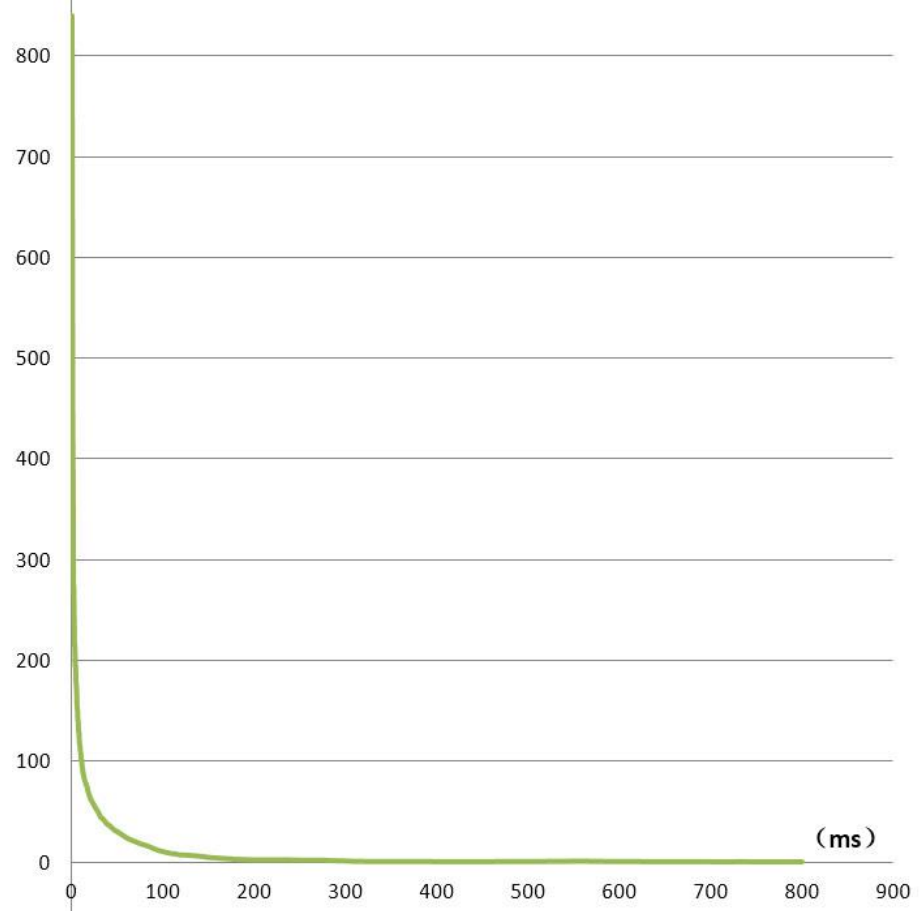

Fig. 4 Sampling signal map

The sampling points were analyzed and fitted by using the magnetic resonance crosslink density analysis software, and the result is shown in the following table:

Table 1. Nuclear magnetic resonance sampling analysis result

\begin{tabular}{|c|c|c|c|c|}
\hline sample & $\mathrm{T}_{2}(\mathrm{~ms})$ & $\mathrm{A}$ & $\mathrm{B}$ & $\begin{array}{c}\mathrm{N} 0 \\
(\mathrm{~mol} / \mathrm{ml})\end{array}$ \\
\hline $\begin{array}{c}\text { HTPB } \\
\text { binder }\end{array}$ & 15.04 & $74.89 \%$ & $25.11 \%$ & $0.92 \times 10^{-4}$ \\
\hline
\end{tabular}

\section{Conclusion}

Compared with the traditional research methods of solid propellant, nuclear magnetic resonance method has the following three advantages:

(1) Non-destructive testing, which means that nuclear magnetic resonance can be directly applied to the equipment installed in the detection, and not to make any damage to the weapons;

(2) The sample can be reused, which can greatly reduce the waste of the sample during the test; 
(3) Fast detection speed, a test only takes about ten minutes, can effectively save the experiment time.

As the equipment service time increases, the service life of the missile weapon system's accurate forecast is particularly important, and the life of the main evaluation criteria is the length of the service life of engine. If you can find a relevance to the network structure and mechanical properties of propellant, the crosslink density of propellant or the size of the transverse relaxation time can be turned as an important evaluation basis, which tell mechanical performance is good or bad for the life of the engine evaluation, providing a new way of thinking.

Although the nuclear magnetic resonance (NMR) method has many advantages, there are still many technical problems to overcome as a kind of new method. Nuclear magnetic resonance analysis software, for example, whose calculation model is created according to the rubber structure, can't completely suitable for propellant; There is no uniform magnetic field and completely accurate RF pulse, only through technology improve the signal-to-noise ratio and resolution of equipment, the test precision will improve. Along with the advance of technology and the deepening of the research, the NMR method is expected to become the new direction for propellant research.

\section{References}

[1].Linfa Hou. Composite Solid Propellant [M]. Beijing:China Space Press, 1994.

[2].Chen Rongsheng,Jian Zheng,Zhongnian Xiong, et al. Theoretical Calculation Method for Structural Parameters of Chemical Crosslinked PU Elastomers [J].The Journal of Explosives and Propellants. Vol. 19 (1996) No. 1, p. 35-42.

[3]. Weina Bi:Study on the Crosslink-Density and Dynamic Properities of NR Compound and Vulcanizates[D](Master,Qingdao University of Science and Technology,China,2008).

[4]. He Q H, Richter W, Vathyam S, et al. Intermolecular Multiple-Quantum Coherences and Cross Correlations in Solution Nuclear Magnetic Resonance[J]. Journal of Chemistry Physics. Vol. 98 (1993) No. 9, p. 6779-6800.

[5]. Warren W S, Richter W,Andreotti A H, et al. Generation of Impossible Cross-Aks Between Bulk Water and Biomolecules in Solution NMR[J]. Science. Vol. 262 (1993) No. 5142, p. 2005-2009.

[6]. Wang Dongyun. Advances in Nuclear Magnetic Resonance Technology and Applications [J]. Science and Technology Information (Academic Research). (2008) No. 27, p. 353-354.

[7]. Haibiao Cui,Fan Lu,Xin Li, et al. D_T_2 Review on the Development of Two-dimensional Nuclear Magnetic Resonance Logging Technology [J]. Petrochemical applications. Vol. 35 (2016) No. 3, p. 1-5.

[8]. Jielin Li,Keping Zhou, Yamin Zhang, et al. Experimental Study on Freezing and Thawing Damage of Rock Pore Structure Based on NMR Technology [J]. Journal of Rock Mechanics and Engineering. Vol. 31 (2012) No. 6, p. 1208-1214.

[9]. Fangfang Wang,Hai Chang,Linjun Zhang, et al. Research on Crosslink-Density Test Method and Application in Explosive and Explosive [J]. The Journal of Explosives and Propellants. Vol. 39 (2016) No. 2, p. 8-15.

[10]. Shouyuan Zhao, Yuanyuan Wang,Rongchun Zhang, et al. Study on Structure and Kinetics of Nanocomposite Gels by Low-field Solid NMR [J].Journal of Spectroscopy. Vol. 31 (2014) No. 2, p. $172-184$.

[11]. Lin Jia,Wuxi Xie,Jiaojiao Du, et al. Study on the Cracking Reaction of Biphenyl Bismuth to BAMO-THF / TDI Binder by LF-NMR[J].The Journal of Explosives and Propellants. Vol. 38 (2015) No. 1, p. 69-73.

[12]. Lin Jia, Wuxi Xie, Jiaojiao Du, et al. Study on the Effect of Burning Rate Catalyst on Propellant Curing Reaction by LF-NMR [J]. Solid Rocket Technology. Vol. 38 (2015) No. 5, p. 697-706.

[13]. Minchang Wang,Gao Zhang,Min Xu, et al. Study on the Determination of a New Liquid Propellant [J]. Energetic Materials. Vol. 15 (2007) No. 4, p. 404-415. 
[14]. Damadian R. Tumor Detection by Nuclear Magnetic Resonance[J]. Science.(2008) No. 171, p. 1151.

[15]. Donglin Zu,Jiahong Gao, et al. Nuclear Magnetic Resonance Imaging-Physical Principles and Methods [M]. Beijing: Peking University Press,2014.9.

[16]. Gould. R. F. .Propellant Manufacture,Hazards, and Testing[J].Advances in Chemistry Series 88,American Chemical Society, 1969.

[17]. Yining Li, et al. Performance and Principle of Solid Propellant [M]. National University of Defense Science and Technology,1987,p.173 306.

[18]. Yan Xiao:Study on Thermal Oxidation of Natural Rubber Vulcanized [D](Master, Northwestern Polytechnical University,China,2006).

[19]. Wei Zhang, Xuezhong Fan,Caining Yang, et al. Determination of Crosslink-Density of NEPE Propellant by Swelling Method [J].Journal of Explosives and Propellants. Vol. 31 (2008) No. 5, p. 51-55.

[20]. Oikawa H,Murakami K. Some Comments on the Swelling Mechanism of Rubber Vulcanizates [J]. Rubber Chemistry and Technology. Vol. 60 (1987) No. 4, p. 579-590.

[21]. Eisele U. Introduction to Polymer Physics [M]. New York: Springer-Verlag, 1990.

[22]. Ming Lu, YonglaiSong, QifengYuan, et al. Improvement and Application of Elastomer Crosslinking Density Tester [J]. Energetic Materials. Vol. 7 (1999) No. 3, p. 141-144.

[23]. Hill L w. Calculation of Crosslink-Density in Short Chain Networks[J]. Progress in Organic Coatings. (1997) No. 31, p. 235-243.

[24]. Mareanukro H M,Eby R K,Scavuzzo R J, et al. Use of Atomic Force Microscope as a Nanoindenter to Characterize Elastomers Rubber[J]. Rubber Chemistry and Technology. Vol. 73 (2000) No. 5, p. 912-925.

[25]. Zuoling Wang. Rubber Crosslink-Density and Determination Method [J]. World Rubber Industry. Vol. 25 (1998) No. 4, p. 41-46.

[26]. Fei Zhao,Ping Zhang,Shugao Zaho, et al. Effect of Sulfur Content on Crosslinking Density and Structure of Natural Rubber by Nuclear Magnetic Resonance [J]. Synthetic Rubber Industry. Vol. 31 (2008) No. 2, p. 113-117. 